\title{
A GALERKIN SOLUTION TO A REGULARIZED CAUCHY SINGULAR INTEGRO-DIFFERENTIAL EQUATION
}

\author{
BY \\ JAY I. FRANKEL \\ Mechanical and Aerospace Engineering Dept., University of Tennessee, Knoxville, Tennessee
}

Abstract. This paper presents a Galerkin approach for solving a regularized version of the Cauchy singular, linear integro-differential equation

$$
\frac{d \Theta}{d x}(x)-f(x)=\lambda f_{y=0}^{1} \frac{\Theta(y)}{x-y} d y, \quad x \in(0,1),
$$

subject to $\Theta(0)=\Theta(1)=0$. This equation has appeared in both combined infrared gaseous radiation and molecular conduction, and elastic contact studies. A regularized formulation is produced which suggests the use of an expansion technique where the orthogonal basis functions are chosen as the Chebychev polynomials of the first kind. Accurate results, requiring a minimal computational cost, are formally documented and compared to a purely numerical solution.

1. Introduction. The mathematical formulation of physical phenomena often involves Cauchy-type (or more severe) singular integral equations. Integral and integrodifferential equations containing strongly singular kernels appear in studies involving elastic contact [1], stress analysis [1], fracture mechanics [2-4], airfoil theory [5-10], and combined infrared radiation and molecular conduction [11, 12]. Owing to their common appearance in practice, there exists a growing need to develop accurate approximate analytical and numerical solutions to a large variety of singular integral and integro-differential equations. Simulation techniques that take advantage of recent hardware and software developments are particularly appealing amid the rapidly changing computational environment.

Over the past thirty years, substantial progress has been made in developing innovative approximate analytical and purely numerical solutions to a large class of singular integral equations. The books edited by Golberg [13, 14] are particularly enlightening and should be consulted since they contain fairly extensive literature surveys on both approximate analytical and purely numerical techniques. The interested reader should also consult the fine expositions by Linz [15], Baker [16], Delves and Mohamed [17], and Atkinson [18] for numerical methods, and consult the books 
by Tricomi [19], Hochstadt [20], Green [21], and Porter and Stirling [22] for information concerning analytical solution methods.

In formulating physical problems, it is worth noting that in many instances the initial formulation may not lend itself easily to approximation [23, 24]. Thus, it may be necessary to recast the original formulation into a form conducive to approximation. This preconditioning often permits additional interpretation and insight into choosing a well-suited numerical method. If analytical preconditioning is not performed, a less satisfactory but more expedient brute force technique is usually opted for, generally yielding marginal results. The present work illustrates that analytic preconditioning leads to a natural approximation process that requires little computational cost.

This paper uses a classical expansion method for solving a linear, Cauchy singular integro-differential equation. Cess and Tiwari [11] derived

$$
\mu \frac{d \Theta}{d x}(x)-f(x)=\lambda f_{y=0}^{1} \frac{\Theta(y)}{x-y} d y, \quad x \in(0,1),
$$

where

$$
f(x)=-\left(x-\frac{1}{2}\right)
$$

with the auxiliary conditions

$$
\Theta(0)=\Theta(1)=0 .
$$

This mathematical formulation was originally developed in the context of combined gaseous infrared radiation and molecular conduction in a plane-parallel geometry. Due to physical symmetry [11], we know $\Theta(x)=\Theta(1-x)$. Here $\Theta(x)$ represents the unknown temperature, $x$ represents the spatial variable, and $\lambda$ is a constant composed of several physical properties. Equations (1) represent the large path length limit to a more general equation presented in [11] (when $\mu=1$ ). Here $f$ denotes integration in the Cauchy principal value sense. We suppose that $\Theta(x), x \in[0,1]$ is continuous and that the derivative $\Theta^{\prime}(x)$ exists and is continuous on the interval $x \in(0,1)$. Then $f_{y=0}^{1} \Theta(y)(x-y)^{-1} d y$ exists in the principal value sense. Cess and Tiwari [11] developed a purely numerical solution to Eq. (1) for $\Theta(x)$. Unfortunately, no details were furnished describing their numerical implementation.

A similar equation to that expressed by Eq. (1a) has also appeared in the study of elastic contact [1]. Sankar et al. [1] developed a power series solution method that included a term that moderated the solution tendencies at the endpoints. If $\mu=0$ in Eq. (1a), then the derivative term vanishes and the classical airfoil equation is recovered. Peters $[25,26]$ developed a simple yet insightful solution method to the airfoil equation based on reducing the airfoil equation to the solution of an Abel integral equation. More recently Golberg [13, 14], and Porter and Stirling [22] have expounded on the merits of Peters' approach. In fracture mechanics [27, 28], Cauchytype kernels (and stronger, i.e., $\left.(x-y)^{-\alpha}, \alpha>1\right)$ often arise. Kaya and Erdogan [27, 28] have reported several interesting findings based on the evaluation of finite-part integrals (i.e., in the sense of Hadamard integration). Additionally, noteable papers (too many to cite) by Ioakimidis $[29,30]$ have considered numerous approximate 
analytic and purely numeric methods for resolving Fredholm integral equations with Cauchy-type singularities.

The present work describes a Galerkin approach for solving Eq. (1) for the case when $\mu=1$. The approach begins by transforming the initial integro-differential equation into an alternative form in which the unknown function $\Theta(x)$ is receptive to approximation by an expansion technique. The basis functions chosen for the expansion are based on Chebychev polynomials. This regularized form permits the use of several integral and algebraic relations and the inclusion of the orthogonality property associated with Chebychev polynomials for determining the unknown expansion coefficients of the basis functions. Results indicate that the approach yields accurate results with a minimal computational cost.

This paper is divided into three main sections. Section 2 presents the alternative formulation based on Peters' notion $[25,26]$ of the simplifying operator. Use of this concept allows us to transform the original integro-differential equation into a regularized form that is conducive to approximation by an orthogonal series representation. Section 3 describes the Chebychev series expansion and describes the Fourier method used in determining the unknown expansion coefficients. Section 4 presents some representative numerical results.

2. Formulation. In this section, we present a regularized form of Eq. (1). The approach taken here uses the concept proposed by Peters $[25,26]$ in studying the airfoil equation. Potter and Stirling [22] have coined the phrase "simplifying operator" to denote the effect of the transformation.

Letting $\mu=1$ and following the well-documented approach offered by Peters [25, 26], we arrive at the regularized form

$$
\Theta(x)=\frac{C_{0}}{\pi \sqrt{x} \sqrt{1-x}}+\frac{1}{\lambda \pi^{2}} f_{z=0}^{1} \frac{d z}{z-x} \frac{\sqrt{z} \sqrt{1-z}}{\sqrt{x} \sqrt{1-x}}\left[\frac{d \Theta}{d z}(z)-f(z)\right],{ }_{x \in(0,1),}
$$

where $C_{0}$ is given by

$$
C_{0}=\int_{y=0}^{1} \Theta(y) d y .
$$

An alternative expression for the constant $C_{0}$ can be developed by first multiplying Eq. (2a) by $\sqrt{x} \sqrt{1-x}$ and then following up by evaluating the resulting form at $x=0$. Doing so, we arrive at

$$
C_{0}=-\frac{1}{\lambda \pi} \int_{z=0}^{1} \sqrt{\frac{1-z}{z}}\left[\frac{d \Theta}{d z}(z)-f(z)\right] d z .
$$

Next, we substitute Eq. (3) into Eq. (2a) for $C_{0}$ to obtain

$$
\Theta(x)=\frac{1}{\lambda \pi^{2}} f_{z=0}^{1}\left[\frac{d \Theta}{d z}(z)-f(z)\right] \sqrt{\frac{x(1-z)}{z(1-x)}} \frac{d z}{z-x}, \quad x \in(0,1) .
$$

We shall refer to Eq. (2a) as our Chebychev form while we refer to Eq. (4) as our Jacobi form. The rationale for this terminology will become clear in the next section. 
It is interesting to note that if we substitute Eq. (2b) into the left-hand side of Eq. (3) for $C_{0}$ and substitute Eq. (1a) into the right-hand side of Eq. (3), we find

$$
\int_{z=0}^{1} \Theta(z) d z=-\frac{1}{\pi} \int_{z=0}^{1} \sqrt{\frac{1-z}{z}}\left[f_{y=0}^{1} \frac{\Theta(y)}{z-y} d y\right] d z,
$$

or upon interchanging the orders of integration, we arrive at

$$
\int_{z=0}^{1} \Theta(z) d z=-\frac{1}{\pi} \int_{y=0}^{1} \Theta(y)\left[f_{z=0}^{1} \sqrt{\frac{1-z}{z}} \frac{d z}{z-y}\right] d y,
$$

thereby identifying the value of the inner integral as

$$
f_{z=0}^{1} \sqrt{\frac{1-z}{z}} \frac{d z}{z-y}=-\pi
$$

Additionally, if we multiply Eq. (2a) by $\sqrt{x} \sqrt{1-x}$ and evaluate the resulting expression at $x=1$, and make use of $\Theta(1)=0$, we obtain yet another expression for $C_{0}$, namely

$$
C_{0}=\frac{1}{\lambda \pi} \int_{z=0}^{1} \sqrt{\frac{z}{1-z}}\left[\frac{d \Theta}{d z}(z)-f(z)\right] d z
$$

Similar logic can be used to show that

$$
f_{z=0}^{1} \sqrt{\frac{z}{1-z}} \frac{d z}{z-y}=\pi
$$

which is identical to the contour integration result reported by $\mathrm{Lu}$ [31].

In closing this section, we state some alternative formulations. The first two forms are based on simple calculus manipulations that are intended to reduce the severity of the singularity. Clearly, an alternative formulation for Eq. (1a) can be expressed as

$$
\frac{d \Theta}{d x}(x)-f(x)=\lambda \int_{y=0}^{1} \frac{d \Theta}{d y}(y) \log |x-y| d y, \quad x \in[0,1],
$$

where $\Theta(x)$ can be recovered by direct integration, namely

$$
\Theta(x)=\int_{y=0}^{x} \frac{d \Theta}{d y}(y) d y, \quad x \in[0,1],
$$

since $\Theta(0)=0$. If one desires a formulation in terms of $\Theta(x)$ directly, one can express Eq. (1a) in the alternative form

$$
\Theta(x)=g(x)+\lambda \int_{y=0}^{1} \Theta(y) \log \left|\frac{x-y}{y}\right| d y, \quad x \in[0,1],
$$

where

$$
g(x)=\int_{y=0}^{x} f(y) d y .
$$

Both Eqs. (7a) and (8a) permit the use of product integration [13, 15-18, 24], and singularity subtraction $[13,17,18,24]$. 
As an alternative to "decreasing" the order, as illustrated by Eq. (8a), we present the contrasting notion of "increasing" the order. To illustrate this concept, we begin by taking the derivative of Eq. (7a) with respect to $x$ to get

$$
\frac{d^{2} \Theta}{d x^{2}}(x)=\frac{d f}{d x}(x)+\lambda \int_{y=0}^{1} \frac{d \Theta}{d y}(y) \frac{d y}{x-y}, \quad x \in(0,1) .
$$

In operator (or symbolic) form, we can express Eq. (1a) when $\mu=1$, and Eq. (9) as

$$
\begin{aligned}
\Theta^{\prime} & =f+\lambda K \Theta, \\
\Theta^{\prime \prime} & =f^{\prime}+\lambda K \Theta^{\prime},
\end{aligned}
$$

respectively. Substituting Eq. (10a) into Eq. (10b) for $\Theta^{\prime}$ yields

$$
\boldsymbol{\Theta}^{\prime \prime}=f^{\prime}+\lambda K f+\lambda^{2} K^{2} \Theta
$$

or explicitly

$$
\begin{aligned}
\frac{d \Theta^{2}}{d x^{2}}(x)=\frac{d f}{d x}(x)+\lambda f_{y=0}^{1} \frac{f(y)}{x-y} d y+\lambda^{2} f_{z=0}^{1} \frac{d z}{x-z} f_{y=0}^{1} \Theta(y) \frac{d y}{z-y}, & x \in(0,1),
\end{aligned}
$$

subject to $\Theta(0)=\boldsymbol{\Theta}(1)=0$. Using the Hardy-Poincaré-Bertrand formula [19] reduces Eq. (10d) to

$$
\frac{d \Theta^{2}}{d x^{2}}(x)+\lambda^{2} \pi^{2} \Theta(x)=h(x)+\lambda^{2} \int_{y=0}^{1} M(x, y) \Theta(y) d y, \quad x \in(0,1),
$$

where

$$
h(x)=\frac{d f}{d x}(x)+\lambda f_{y=0}^{1} \frac{f(y)}{x-y} d y,
$$

and with

$$
M(x, y)=\frac{1}{x-y} \log \left[\frac{x(1-y)}{y(1-x)}\right]=M(y, x) .
$$

Notice that $M(x, x)=[x(1-x)]^{-1}$. It is interesting to note that one can approximate the kernel $M(x, y)$ in a separable form [32]. Conversion of Eq. (11a) into an equivalent integral equation can be accomplished with the aid of the Green's function method. Using this formulation, one can develop a solution based on solving for the moments of the unknown function. This approach has been explored and some results have been documented. it should be noted that the approach presented in the next section appears to be the most acceptable solution method studied by the present author.

3. Method of solution. This section is dedicated to developing an approximate solution of Eq. (2) subject to the auxiliary condition expressed by Eq. (1b). Before proceeding further, it is convenient to map the physical domain from $x \in[0,1]$ to $\eta \in[-1,1]$, thereby arriving at

$$
\Theta(\eta) \sqrt{1-\eta^{2}}=\frac{1}{\pi} \int_{\xi=-1}^{1} \Theta(\xi) d \xi+\frac{2}{\lambda \pi^{2}} f_{\xi=-1}^{1} \frac{\sqrt{1-\xi^{2}}}{\xi-\eta}\left[\frac{d \Theta}{d \xi}(\xi)-\frac{f\left(\frac{\xi+1}{2}\right)}{2}\right] d \xi,
$$


subject to

$$
\Theta(-1)=\Theta(1)=0 .
$$

Here $f\left(\frac{\xi+1}{2}\right)$ reduces to $-\xi / 2$. Therefore, Eq. (12a) can be expressed as

$$
\begin{aligned}
\Theta(\eta) \sqrt{1-\eta^{2}}-\frac{1}{\pi} \int_{\xi=-1}^{1} \Theta(\xi) d \xi & +\frac{2}{\lambda \pi^{2}} \int_{\xi=-1}^{1} \frac{\sqrt{1-\xi^{2}}}{\eta-\xi} \frac{d \Theta}{d \xi}(\xi) d \xi \\
& =-\frac{1}{2 \lambda \pi^{2}} f_{\xi=-1}^{1} \frac{\xi \sqrt{1-\xi^{2}}}{\eta-\xi} d \xi, \quad \eta \in(-1,1) .
\end{aligned}
$$

The manipulations leading to Eq. (13) illustrate the merit of analytic preconditioning since this new formulation naturally suggests the use of a Chebychev series expansion for the unknown function $\Theta(\eta)$. Let $T_{m}(\eta)=\cos \left[m\left(\cos ^{-1} \eta\right)\right]$, $\eta \in[-1,1], m=0,1, \ldots$ denote the Chebychev polynomials of the first kind while $U_{m}(\eta)=\sin \left[(m+1)\left(\cos ^{-1} \eta\right)\right] / \sin \left(\cos ^{-1} \eta\right), m=0,1, \ldots$ denote the Chebychev polynomials of the second kind. It is well known that $\left\{T_{m}(\eta)\right\}, m=0,1, \ldots$ form an orthogonal sequence of functions with respect to the weight function $\left(1-\eta^{2}\right)^{-1 / 2}$ while $\left\{U_{m}(\eta)\right\}, m=0,1, \ldots$ form an orthogonal sequence of functions with respect to the weight function $\left(1-\eta^{2}\right)^{1 / 2}$.

It appears reasonable to attempt a series expansion to $\Theta(\eta)$ in Eq. (13) in terms of Chebychev polynomials of the first kind. This choice is not arbitrary since one can identify a portion of the integrand as the weight function associated with $T_{m}(\eta)$. It now becomes apparent why we have coined the phrases Chebychev and Jacobi forms when speaking of Eq. (2a) and Eq. (4), respectively.

We assume that the function $\Theta(\eta)$ has an expansion of the form (other choices also exist that are acceptable)

$$
\boldsymbol{\Theta}(\eta)=\sum_{m=0,2, \ldots}^{\infty} a_{m} T_{m}(\eta)-1, \quad \eta \in(-1,1),
$$

where $T_{m}(\eta)$ represents the $m$ th Chebychev polynomial of the first kind. The unknown expansion coefficients $a_{m}, m=0,2, \ldots$ are to be determined by some means, say either by a collocation or Galerkin method. Notice that only even powers of the Chebychev polynomials are preserved. This is done in light of the physical fact (i.e., symmetry) that $\Theta(\eta)=\Theta(-\eta)$. Additionally, we impose the auxiliary condition shown in Eq. $(12 b)$, namely $\Theta( \pm 1)=0$. Imposing this constraint yields

$$
\sum_{m=0,2, \ldots}^{\infty} a_{m}=1
$$

since $T_{m}( \pm 1)=1, m=0,2, \ldots$ [33]. Thus, we can express Eq. (14a) as

$$
\Theta(\eta)=\sum_{m=0,2, \ldots}^{\infty} a_{m}\left[T_{m}(\eta)-1\right], \quad \eta \in[-1,1] .
$$

The rationale for this auxiliary profile condition is apparent due to the integrodifferential form of the equation. The derivative of Eq. (14c) with respect to $\eta$ 
becomes

$$
\frac{d \Theta}{d \eta}(\eta)=\sum_{m=2,4, \ldots}^{\infty} m a_{m} U_{m-1}(\eta), \quad \eta \in[-1,1],
$$

since $\frac{d T_{m}}{d \eta}(\eta)=m U_{m-1}$ [33]. Here $U_{m}(\eta)$ denotes the $m$ th Chebychev polynomial of the second kind. Substituting Eq. (14c) and Eq. (15) into Eq. (13) yields

$$
\begin{aligned}
\sqrt{1-\eta^{2}} \sum_{m=0,2, \ldots}^{\infty} a_{m} & {\left[T_{m}(\eta)-T_{0}(\eta)\right]-\frac{1}{\pi} \sum_{m=0,2, \ldots}^{\infty} a_{m} \int_{\xi=-1}^{1}\left[T_{m}(\xi)-T_{0}(\xi)\right] d \xi } \\
& +\frac{2}{\lambda \pi^{2}} \sum_{m=2,4, \ldots}^{\infty} m a_{m} f_{\xi=-1}^{1} \frac{\sqrt{1-\xi^{2}}}{\eta-\xi} U_{m-1}(\xi) d \xi \\
& =-\frac{1}{4 \lambda \pi^{2}} f_{\xi=-1}^{1} \frac{\sqrt{1-\xi^{2}}}{\eta-\xi} U_{1}(\xi) d \xi,
\end{aligned}
$$

where we have made the substitutions $1=T_{0}(\eta)=T_{0}(\xi)$ and $U_{1}(\xi)=2 \xi$.

For convenience, we state some well-known algebraic and integral relations associated with the Chebychev polynomials.

Orthogonality property [33].

$$
\int_{\xi=-1}^{1}\left(1-\xi^{2}\right)^{-1 / 2} T_{m}(\xi) T_{n}(\xi) d \xi= \begin{cases}0, & m \neq n, \\ \pi, & m=n=0, \\ \frac{\pi}{2}, & m=n>0 .\end{cases}
$$

Closed-form integral relations $[27,28]$.

$$
\begin{aligned}
& f_{\xi=-1}^{1} \frac{U_{n}(\xi) \sqrt{1-\xi^{2}}}{\xi-\eta} d \xi=-\pi T_{n+1}(\eta), \quad n=0,1, \ldots \\
& \int_{\xi=-1}^{1} T_{n}(\xi) d \xi= \begin{cases}\frac{2}{1-n^{2}}, & n=0,2, \ldots \\
0, & n=1,3, \ldots .\end{cases}
\end{aligned}
$$

Algebraic relation [34].

$$
T_{m}(\xi) T_{n}(\xi)=\frac{1}{2}\left[T_{m+n}(\xi)+T_{|m-n|}(\xi)\right], \quad m=0,1, \ldots, n=0,1, \ldots
$$

Three-term recurrence relation $[33,35]$.

$$
\begin{aligned}
T_{n+1}(\xi)-2 \xi T_{n}(\xi)+T_{n-1}(\xi)=0, & n=1,2, \ldots, \\
U_{n+1}(\xi)-2 \xi U_{n}(\xi)+U_{n-1}(\xi)=0, & n=1,2, \ldots .
\end{aligned}
$$

The above expressions contain all the necessary ingredients for determining the unknown expansion coefficients $a_{m}, m=0,2, \ldots$.

Using Eq. (18), Eq. (16) reduces to

$$
\begin{aligned}
& \sqrt{1-\eta^{2}} \sum_{m=0,2, \ldots}^{\infty} a_{m}\left[T_{m}(\eta)-T_{0}(\eta)\right]-\frac{1}{\pi} \sum_{m=0,2, \ldots}^{\infty} a_{m}\left[\frac{2}{1-m^{2}}-2\right] \\
& \quad+\frac{2}{\lambda \pi} \sum_{m=2,4, \ldots}^{\infty} m a_{m} T_{m}(\eta)=-\frac{1}{4 \lambda \pi} T_{2}(\eta), \quad \eta \in[-1,1] .
\end{aligned}
$$


The salient property required for determining the unknown expansion coefficients is derived from the orthogonality relation shown in Eq. (17). Thus, our approach is to follow a classical Fourier technique for determining the unknown expansion coefficients. Dividing Eq. (21) by $\sqrt{1-\eta^{2}}$ and then operating on the result with

$$
\int_{\eta=-1}^{1} T_{n}(\eta) d \eta
$$

we arrive at

$$
\begin{gathered}
\sum_{m=0,2, \ldots}^{\infty} a_{m} \int_{\eta=-1}^{1}\left[T_{m}(\eta)-T_{0}(\eta)\right] T_{n}(\eta) d \eta-\frac{1}{\pi} \sum_{m=0,2, \ldots}^{\infty} a_{m}\left[\frac{2}{1-m^{2}}-2\right] \int_{\eta=-1}^{1} \frac{T_{n}(\eta)}{\sqrt{1-\eta^{2}}} d \eta \\
\quad+\frac{2}{\lambda \pi} \sum_{m=2,4, \ldots}^{\infty} m a_{m} \int_{\eta=-1}^{1} \frac{T_{m}(\eta) T_{n}(\eta)}{\sqrt{1-\eta^{2}}} d \eta \\
=-\frac{1}{4 \lambda \pi} \int_{\eta=-1}^{1} \frac{T_{2}(\eta) T_{n}(\eta)}{\sqrt{1-\eta^{2}}} d \eta, \quad n=0,2, \ldots, \quad \eta \in[-1,1], \lambda>0 .
\end{gathered}
$$

Making use of the previously reported closed-form integral relations, Eq. (22) then gives rise to the remarkably compact form

$$
\sum_{m=0,2, \ldots}^{\infty} a_{m} c_{m n}=-\frac{1}{8 \lambda} \delta_{2, n}, \quad n=0,2, \ldots,
$$

where $c_{m n}$ is defined as

$$
\begin{aligned}
c_{m n}=\frac{1}{1-(m+n)^{2}}+\frac{1}{1-(m-n)^{2}}-\frac{2}{1-n^{2}}+\frac{n}{\lambda} \delta_{m, n}, \\
\quad m=0,2, \ldots, n=0,2, \ldots,
\end{aligned}
$$

and where $\delta_{m, n}$ represents the Kronecker delta. Thus, Eq. (23a) leads to an infinite system of linear algebraic equations for the unknown expansion coefficients $a_{m}$, $m=0,2, \ldots$. It is clear from viewing the above system of equations that $c_{0 n}=0$, $n=0,2, \ldots$. Thus in order to assure a unique solution for the unknown Chebychev coefficients, we replace the first equation in the system shown in Eq. (23a), i.e., $n=0$, by the auxiliary profile condition expressed in Eq. (14b).

Practically speaking, we must truncate the series representation shown in Eq. (14c) after some finite number of terms, say $N$ (even), such that

$$
\Theta(\eta) \cong \Theta^{N}(\eta)=\sum_{m=0,2, \ldots}^{N} a_{m}\left[T_{m}(\eta)-1\right], \quad \eta \in[-1,1] .
$$

Thus, we are left with determining only $[(N / 2)+1]$ coefficients. We refer to $\Theta^{N}(\eta)$ as the approximate solution to $\Theta(\eta)$ in terms of a finite Chebychev series. Results from several numerical experiments are presented in the next section. 
4. Results and discussion. In this section, we present some representative numerical results illustrating the effectiveness of the regularized formulation/Galerkin solution discussed in the preceding sections. Being a formal presentation, convergence $[7,8,30]$ and accuracy are demonstrated by empirical means. A further study delving into theoretical issues is presently under consideration.

The physical parameter $\lambda$, known as the radiation-conduction number for the large path length limit, represents the single parameter of the dimensionless system shown in Eq. (1). As $\lambda$ increases, the effect of molecular conduction diminishes relative to infrared radiation. Also, the contribution of the integral term tends to increase as $\lambda$ increases. Through the approximation process, we must also consider the effect of the number of terms $(N / 2+1)$ retained in the expansion on the accuracy of the series representation for the unknown function $\Theta(\eta)$. The function $\Theta(\eta)$ can be reconstructed through Eq. (24) once the expansion coefficients $a_{m}, m=$ $0,2, \ldots, N$ have been resolved. This approximation process naturally lends itself to a massively parallel computation since one can obtain numerical results for $\Theta(\eta)$ at some predefined set of spatial locations in a parallel fashion.

Table 1 illustrates the effect of coupling among the expansion coefficients $a_{m}$, $m=0,2, \ldots, N$ as a function of the radiation-conduction number $\lambda$. Columns 35 contain only the first eleven (dominant) terms. Clearly, as $\lambda$ increases, the strength of the coupling among the corresponding coefficients becomes more pronounced. As $\lambda$ increases, we observe that the leading term becomes even more dominant relative to the next term. Additionally, as $\lambda$ increases, we see that the coefficients $a_{m}$, $m=0,2, \ldots, N$ decrease in magnitude at a more moderate rate. For $\lambda=0.1$, we find $a_{100}=O\left(10^{-11}\right)$; for $\lambda=1,10$ we find that $a_{100}=O\left(10^{-10}\right)$.

Tables 2 and 3 present a comparison between the approximate solution based on Eq. (2a) that was formally programmed using Eqs. (23) and (24) to that of the purely numerical solution based on Eq. (7). The purely numerical solution was obtained with the aid of singularity subtraction $[13,24]$ and simple trapezoidal integration [36].

Table 2 displays results from the approximate solution as a function of $\lambda$ at six spatial locations $\eta \in[0,1]$ for different values of $N$. Here $(N / 2+1)$ denotes the actual number of terms retained in the series representation shown in Eq. (24). Only half of the physical domain is displayed due to symmetry, $\boldsymbol{\Theta}(\eta)=\boldsymbol{\Theta}(-\eta)$. As indicated by this table, as $N$ increases, convergence to at least 5 places of accuracy appears to occur for the three cases illustrated $(\lambda=0.1,1,10)$. Knowing the behaviour of the coefficients, as depicted in Table 1 (see p. 254), and since $\left|T_{m}(\eta)\right| \leq 1$, $m=0,2, \ldots, N$, we get a qualitative feel that convergence is close at hand. Numerical results for the approximate solution were obtained using both an IBM-XT with a math coprocessor and an Ardent Titan II Graphics Supercomputer. Complete simulations (CPU time and I/O) required less than 8 seconds per test case on the IBM while complete simulations required less than 0.21 seconds on the Titan II (single processor, i.e., serial mode). The developed computer code was not optimized in any manner. The results presented in Tables 2 and 3 (see pp. 255, 256) were obtained using the Ardent Titan II Graphics Supercomputer. The generated results shown in 


\begin{tabular}{|c|c|c|c|c|}
\hline \multicolumn{5}{|c|}{$\begin{array}{l}\text { Expansion coefficients for finite } \\
\text { Chebychev series, } a_{j}, j=0,2, \ldots, N\end{array}$} \\
\hline$\lambda$ & $j$ & $N=20$ & $N=40$ & $N=80$ \\
\hline & 0 & $0.1058220 E+01$ & $0.1058220 E+01$ & $0.1058220 E+0$ \\
\hline & 2 & $0.5786490 E-01$ & $-0.5786490 E-01$ & $-0.5786490 E-01$ \\
\hline & 4 & $-0.3217863 E-03$ & $-0.3217860 E-03$ & $-0.3217860 E-03$ \\
\hline & 6 & $-0.2560451 E-04$ & $-0.2560443 E-04$ & $-0.2560443 E-04$ \\
\hline & 8 & $-0.5216013 E-05$ & $-0.5215983 E-05$ & $-0.5215981 E-05$ \\
\hline & 10 & $-0.1600168 E-05$ & $-0.1600155 E-05$ & -0.1600 \\
\hline & 12 & $-0.6211032 E-06$ & $-0.6210982 E-06$ & $-0.6210976 E-06$ \\
\hline & 14 & $-0.2814815 E-06$ & $-0.2814814 E-06$ & $-0.2814810 E-06$ \\
\hline & 16 & $-0.1424587 E-06$ & $-0.1424632 E-06$ & $-0.1424629 E-06$ \\
\hline & 18 & $-0.7832737 E-07$ & $-0.7834016 E-07$ & $-0.7834000 E-07$ \\
\hline & 20 & $-0.4590472 E-07$ & $-0.4595899 E-07$ & $-0.4595889 E-07$ \\
\hline & 0 & $036350 E+01$ & $0.1036351 E+01$ & $1 E+01$ \\
\hline & 2 & $-0.3454941 E-01$ & $-0.3454930 E-01$ & -0.345 \\
\hline & 4 & $88 E-02$ & $-0.1549966 E-02$ & -0.15 \\
\hline & 6 & $90 E-03$ & $-0.1889926 E-03$ & -0.1 \\
\hline & 8 & -0 . & $-0.4055730 E-04$ & -0. \\
\hline & 10 & $04 E-04$ & $1 E-04$ & -0 . \\
\hline & 12 & $6 E-05$ & $4 E-05$ & -0 . \\
\hline & 14 & $4 E-05$ & $6 E-05$ & -0.2 \\
\hline & 16 & $4 E-05$ & $347 E-05$ & $8 E-05$ \\
\hline & 18 & $-0.5923305 E-06$ & $-0.5933078 E-06$ & $-0.5932955 E-06$ \\
\hline & 20 & $-0.3429042 E-06$ & $-0.3468224 E-06$ & -0.346 \\
\hline 10 & 0 & $8119 E+01$ & $0.1008120 E+01$ & $0 E+01$ \\
\hline & 2 & $-0.6608189 E-02$ & $-0.6607234 E-02$ & $-0.6607175 E-02$ \\
\hline & 4 & $-0.1026673 E-02$ & $-0.1026240 E-02$ & $-0.1026213 E-02$ \\
\hline & 6 & $-0.2974672 E-03$ & $-0.2972585 E-03$ & $-0.2972450 E-03$ \\
\hline & 8 & $9435 E-03$ & $-0.1058395 E-03$ & $4 E-03$ \\
\hline & 10 & $36 E-04$ & -0.42800 & -0.4 \\
\hline & 12 & $-0.1914318 E-04$ & $-0.1912122 E-04$ & $-0.1911885 E-04$ \\
\hline & 14 & $-0.9299324 E-05$ & $-0.9296901 E-05$ & $-0.9295431 E-05$ \\
\hline & 16 & $-0.4847851 E-05$ & $-0.4863665 E-05$ & $-0.4862725 E-05$ \\
\hline & 18 & $666322 E-05$ & $-0.2710667 E-05$ & $-0.2710053 E-05$ \\
\hline & 20 & $-0.1465065 E-05$ & $-0.1595284 E-05$ & $-0.1594883 E-0$ \\
\hline
\end{tabular}

TABLE 1. First eleven Chebychev expansion coefficients for $\lambda=$ $0.1,1,10$. Here $[(N / 2)+1]$ represents the number of terms retained in the series expansion. 


\begin{tabular}{|l|l|l|l|l|}
\hline \multicolumn{5}{|c|}{$\begin{array}{l}\text { Approximate Solution, Eq. (2a) } \\
\text { for } \Theta(\eta)\end{array}$} \\
\hline$\lambda$ & $\eta$ & \multicolumn{1}{|c|}{$N=40$} & \multicolumn{1}{|c|}{$N=80$} & \multicolumn{1}{|c|}{$N=100$} \\
\hline 0.1 & 1 & 0 & 0 & 0 \\
& 0.8 & 0.04230519 & 0.04230520 & 0.04230520 \\
& 0.6 & 0.07467375 & 0.07467375 & 0.07467375 \\
& 0.4 & 0.09757693 & 0.09757693 & 0.09757693 \\
& 0.2 & 0.1112420 & 0.1112420 & 0.1112420 \\
& 0 & 0.1157850 & 0.1157850 & 0.1157850 \\
& & & & \\
1 & 1 & 0 & 0 & 0 \\
& 0.8 & 0.02809824 & 0.02809828 & 0.02809828 \\
& 0.6 & 0.04718276 & 0.04718280 & 0.04718280 \\
& 0.4 & 0.05984623 & 0.05984626 & 0.05984626 \\
& 0.2 & 0.06712799 & 0.06712802 & 0.06712802 \\
& 0 & 0.06950770 & 0.06950773 & 0.06950773 \\
& & & & \\
10 & 1 & 0 & 0 & 0 \\
& 0.8 & 0.007278492 & 0.007278589 & 0.007278593 \\
& 0.6 & 0.01060173 & 0.01060179 & 0.01060179 \\
& 0.4 & 0.01253844 & 0.01253848 & 0.01253848 \\
& 0.2 & 0.01358764 & 0.01358768 & 0.01358768 \\
& 0 & 0.01392214 & 0.01392220 & 0.01392220 \\
\hline
\end{tabular}

TABLE 2. Approximate solution for $\Theta(\eta)=\Theta(-\eta)$ using the finite Chebychev series representation shown in Eq. (24). Here $[(N / 2)+1]$ denotes the actual number of terms retained in the series expansion. (Note that when $\lambda=10, \Theta^{N}(0.8)=0.007278595$ for $N=$ 150,200 .)

Table 3 took substantially longer run times when compared to the results presented in Table 2.

Results from the numerical solution based on singularity subtraction and trapezoidal integration are shown in Table 3. The number of panels used in the trapezoidal rule is indicated as $M-1$. If the integrand is smooth, it is well known that the global truncation error associated with trapezoidal integration is $O\left(\Delta \eta^{2}\right)$ [36]. However, in the presence of a weakly singular kernel, this error is not anticipated [18]. The global truncation error of the implemented trapezoidal rule can be empirically approximated [18] if one assumes that the results presented in Table $2(N=100)$ are numerically "exact" to the indicated places. In the present study, we found that the global truncation error is $O\left(\Delta \eta^{r}\right)$ where $1.7<r<1.8$ which is in line with our expectations, i.e., $r<2$. As the number of panels in the integration rule is increased, results from the purely numerical solution appear to be approaching the finite Chebychev series results shown in Table 2. 


\begin{tabular}{|c|c|c|c|c|}
\hline \multicolumn{5}{|c|}{$\begin{array}{l}\text { Numerical Solution, Eq. (7a) } \\
\text { for } \Theta(\eta)\end{array}$} \\
\hline$\lambda$ & $\eta$ & $M=51$ & $M=101$ & $M=201$ \\
\hline \multirow[t]{6}{*}{0.1} & 1 & 0 & 0 & 0 \\
\hline & 0.8 & 0.04231206 & 0.04230719 & 0.04230576 \\
\hline & 0.6 & 0.07468139 & 0.07467594 & 0.07467436 \\
\hline & 0.4 & 0.09758491 & 0.09757920 & 0.09757756 \\
\hline & 0.2 & 0.1112502 & 0.1112444 & 0.1112427 \\
\hline & 0 & 0.1157932 & 0.1157874 & 0.1157857 \\
\hline \multirow[t]{6}{*}{1} & 1 & 0 & 0 & 0 \\
\hline & 0.8 & 0.02813853 & 0.02811001 & 0.02810163 \\
\hline & 0.6 & 0.04722369 & 0.04719451 & 0.04718609 \\
\hline & 0.4 & 0.05988672 & 0.05985778 & 0.05984948 \\
\hline & 0.2 & 0.06716808 & 0.0671394 & 0.06713119 \\
\hline & 0 & 0.06954765 & 0.06951906 & 0.06951088 \\
\hline \multirow[t]{6}{*}{10} & 1 & 0 & 0 & 0 \\
\hline & 0.8 & 0.007343878 & 0.00729894 & 0.007284601 \\
\hline & 0.6 & 0.01065554 & 0.01061828 & 0.01060661 \\
\hline & 0.4 & 0.01258667 & 0.01255319 & 0.01254276 \\
\hline & 0.2 & 0.01363321 & 0.01360155 & 0.01359171 \\
\hline & 0 & 0.01396694 & 0.01393582 & 0.01392616 \\
\hline
\end{tabular}

TABLE 3. Results for $\Theta(\eta)$ as obtained by singularity subtraction and trapezoidal integration. Here $M-1$ denotes the number of panels used.

5. Conclusions. This paper demonstrates that analytic preconditioning of the Cess and Tiwari equation, using the method of Peters, allows for the development of an accurate approximate solution based on a finite Chebychev series representation. The tantalizing results offered here illustrate that Peters' approach can be easily extended to Cauchy singular integro-differential equations to produce a formulation highly receptive to approximation by an expansion technique. Extensions to and theoretical issues concerning the presented approach are under consideration. A symbolic (computation) augmentation is also being pursued that will be useful in error analysis.

Acknowledgments. This research was supported by grants provided by the Department of Energy (DE-FG05-92ER25138) and the Florida Solar Energy Center. The author also wishes to thank Mr. George Forbes of Kubota Pacific Inc. (formerly Stardent/Ardent Computers) for continual computer support. 


\section{REFERENCES}

[1] T. S. Sankar, S. V. Hoa, and V. I. Fabrikant, Approximate solution of singular integro-differential equations in elastic contact problems, Internat. J. Numer. Methods Engrg. 18, 503-519 (1982)

[2] F. Erdogan, Approximate solutions of systems of singular integral equations, SIAM J. Appl. Math. 17, 1041-1059 (1969)

[3] F. Erdogan and G. D. Gupta, On the numerical solution of singular integral equations, Quart. Appl. Math. 30, 525-534 (1972)

[4] A. Gerasoulis and R. P. Srivastav, A method for the numerical solution of singular integral equations with a principal value integral, Internat. J. Engrg. Sci. 19, 1293-1298 (1981)

[5] S. R. Bland, The two-dimensional oscillating airfoil in a wind tunnel in subsonic flow, SIAM J. Appl. Math. 18, 830-848 (1970)

[6] J. A. Fromme and M. A. Golberg, Aerodynamics interface effects on oscillating airfoils with controls in ventilated wind tunnels, AIAA J. 18, 417-426 (1980)

[7] M. A. Golberg, The convergence of a collocation method for a class of Cauchy singular integral equations, J. Math. Anal. Appl. 100, 500-512 (1984)

[8] M. A. Golberg and J. A. Fromme, On the $L_{2}$ convergence of collocation for the generalized airfoil equation, J. Math. Anal. Appl. 71, 271-286 (1979)

[9] A. Plotkin and S. S. Dodbele, Slender wing in ground effect, AIAA J. 26, 493-494 (1988)

[10] L. Dragos, Numerical solution of the equation for a thin airfoil in ground effect, AIAA. J. 28, 2132-2134 (1990)

[11] R. D. Cess and S. N. Tiwari, The interaction of thermal conduction and infrared gaseous radiation, Appl. Sci. Res. 20, 25-39 (1969)

[12] E. M. Sparrow and R. D. Cess, Radiation Heat Transfer, McGraw-Hill, New York, 1978

[13] M. A. Golberg, ed., Solution Methods for Integral Equations, Plenum Press, New York, 1979

[14] M. A. Golberg, ed., Numerical Solution of Integral Equations, Plenum Press, New York, 1990

[15] P. Linz, Analytical and Numerical Methods for Volterra Equations, SIAM, Philadelphia, 1985

[16] C. T. H. Baker, The Numerical Treatment of Integral Equations, Clarendon Press, Oxford, 1978

[17] L. M. Delves and J. L. Mohamed, Computational Methods for Integral Equations, Cambridge Univ. Press, Cambridge, 1988

[18] K. E. Atkinson, A Survey of Numerical Methods for the Solution of Fredholm Integral Equations of the Second Kind, SIAM, Philadelphia, 1976

[19] F. G. Tricomi, Integral Equations, Dover, New York, 1985

[20] H. Hochstadt, Integral Equations, Wiley, New York, 1973

[21] C. D. Green, Integral Equation Methods, Barnes and Noble, New York, 1969

[22] D. Porter and D. S. G. Stirling, Integral Equations, Cambridge Univ. Press, Cambridge, 1990

[23] J. I. Frankel, Regularized and preconditioned boundary integral solution to heat transfer in a participating gas flow between parallel plates, Numer. Heat Transfer 19, 105-126 (1991)

[24] J. I. Frankel, Nonlinear heat transfer: Solution of singular, nonlinear integral equations, Engrg. Anal. 8, 231-238 (1991)

[25] A. S. Peters, A note on the integral equation of the first kind with a Cauchy kernel, Comm. Pure Appl. Math. 16, 57-61 (1963)

[26] A. S. Peters, Abel's equation and the Cauchy integral equation of the second kind, Comm. Pure Appl. Math. 21, 51-65 (1968)

[27] A. C. Kaya and F. Erdogan, On the solution of integral equations with strongly singular kernels, Quart. Appl. Math. 45, 105-122 (1987)

[28] A. C. Kaya and F. Erdogan, On the solution of integral equations with strong singularities, in Numerical Solution of Singular Integral Equations (A. Gersoulis and V. Vichnevetsky, eds.), IMACS, 1984, pp. 54-57

[29] N. I. Ioakimidis, On the numerical solution of Cauchy type singular integral equations by the collocation method, Appl. Math. Comp. 12, 49-60 (1983)

[30] N. I. Ioakimidis, Further convergence results for the weighted Galerkin method of numerical solution of Cauchy-type singular integral equations, Math. Comp. 41, 79-85 (1983)

[31] Chien-Ke Lu, A class of quadrature formulas of Chebyshev type for singular integrals, J. Math. Anal. Appl. 100, 416-435 (1984)

[32] J. I. Frankel, unpublished analysis and computations, 1992 
[33] L. C. Andrews, Special Functions of Mathematics for Engineers, 2nd ed., McGraw-Hill, New York, 1992

[34] T. J. Rivlin, The Chebyshev Polynomials, Wiley, New York, 1974

[35] M. Abramowitz and I. A. Stegun, eds., Handbook of Mathematical Functions, Dover, New York, 1972

[36] K. E. Atkinson, An Introduction to Numerical Analysis, 2nd ed., Wiley, New York, 1989 